\title{
O tratamento da Leucemia Mielóide Crônica com mesilato de imatinibe
}

\section{Therapy of Chronic Myeloid Leukemia with imatinib mesylate}

\author{
Vaneuza M. Funke ${ }^{1}$ \\ Daniela C. Setubal ${ }^{2}$ \\ Jefferson Ruiz ${ }^{2}$ \\ Ana Paula Azambuja ${ }^{3}$ \\ Denise H. Lima \\ Terezinha K. Kojo ${ }^{4}$ \\ Ricardo Pasquini ${ }^{5}$
}

\begin{abstract}
O mesilato de imatinibe é atualmente o tratamento de escolha para pacientes com Leucemia mielóide Crônica (LMC) recém-diagnosticados. Desde os primeiros estudos clínicos em 1998 até o estudo IRIS, que comparou o uso em primeira linha de imatinibe com interferon + ara-C, esta droga vem se consolidando em segurança e eficácia. Ainda há, entretanto questionamentos sobre a melhor dose inicial, a identificação dos pacientes que mais se beneficiariam e a melhor abordagem frente a respostas subótimas e resistência. Os principais estudos clínicos publicados com mesilato de imatinibe são revisados no presente artigo, e discutidos sob a perspectiva da realidade brasileira. Rev. bras. hematol. hemoter. 2008; 30(Supl.1):27-31.
\end{abstract}

Palavras-chave: Leucemia Mielóide Crônica; mesilato de imatinibe.

\section{Introdução}

Durante muitas décadas, uma grande variedade de conhecimentos acerca dos eventos moleculares envolvidos no desenvolvimento do câncer humano emergiu. Através do entendimento das vias de sinalização que regulam o crescimento, o ciclo celular e a apoptose, surgiram numerosos alvos para agentes antineoplásicos. O mesilato de imatinibe é atualmente o melhor e mais bem sucedido exemplo da utilização do conhecimento profundo da patogênese molecular de uma neoplasia maligna humana, para o desenvolvimento de uma terapia que tem como alvo um evento envolvido nesta patogênese. Também é a prova definitiva de como a confluência de diversas linhas de investigação pode resultar em avanços significativos para as diversas áreas da ciência médica. ${ }^{1}$ Os excelentes resultados dos estudos clínicos com mesilato de imatinibe revolucionaram o tratamento da Leucemia Mielóide Crônica e tornaram, em pouco tempo, esta droga o tratamento de escolha para pacientes recém-diagnosticados.

\section{Primeiros estudos clínicos com mesilato de imatinibe em Leucemia Mielóide Crônica}

Os primeiros estudos clínicos com o mesilato de imatinibe datam de 1998. No estudo de fase I, foram avaliados 83 pacientes com LMC em fase crônica sem resposta a tratamento prévio com alfa-interferon. Estes pacientes receberam doses de 25-1000 mg ao dia. De 54 pacientes tratados com dose diária de 300 mg ou mais, 53 apresentaram resposta hematológica completa nas primeiras quatro semanas de tratamento. Neste grupo, 29 apresentaram resposta citogenética, incluindo 17 com resposta maior e sete com resposta completa. Doses menores que $300 \mathrm{mg}$ tiveram índices de resposta inferiores, e não são recomendadas. A dose de 400 mg para pacientes passou a ser recomendada na fase crônica da doença. Não foi identificada uma dose máxima tolerada. Os efeitos colaterais foram em sua maioria leves (graus 1 e 2, segundo os critérios de toxicidade do National Cancer Institute). ${ }^{2}$

Os estudos de fase II que rapidamente se sucederam compreenderam pacientes com leucemia mielóide crônica

${ }^{1}$ Médica do Serviço de Transplante de Medula Óssea do Hospital de Clínicas da Universidade Federal do Paraná - HC-UFPR.

Mestre em Hematologia.

${ }^{2}$ Médico(a) do Serviço de Transplante de Medula Óssea do HC-UFPR.

${ }^{3}$ Médica do Serviço de Hematologia do HC-UFPR.

${ }^{4}$ Enfermeira do Serviço de Transplante de Medula Óssea do HC-UFPR.

${ }^{5}$ Professor Titular de Hematologia da Universidade Federal do Paraná - UFPR. Chefe do Serviço de Transplante de Medula Óssea do HC-UFPR.

Correspondência: Vaneuza Araújo Moreira Funke

Rua General Carneiro, 181, $15^{\circ}$ andar

Hospital de Clínicas - Alto da Glória

80060-900 - Curitiba-PR - Brasil

E-mail:vaneuzamf@uol.com.br 
Tabela 1. Resposta hematológica e citogenética ao mesilato de imatinibe: Estudos de Fase II

\begin{tabular}{lccc}
\hline \multicolumn{1}{c}{ Pacientes } & $\begin{array}{c}\text { STI-571 0110 } \\
(\text { Fase crônica })^{3} \\
\mathrm{n}=454\end{array}$ & $\begin{array}{c}\text { STI-571 0109 } \\
(\text { Fase acelerada) } \\
\mathrm{n}=181\end{array}$ & $\begin{array}{c}\text { STI-571 0102 } \\
(\text { Crise blástica })^{5} \\
\mathrm{n}=22\end{array}$ \\
\hline $\begin{array}{l}\text { Resposta Hematológica } \\
\text { Completa }\end{array}$ & $415(91 \%)$ & $125(69 \%)$ & $66(29 \%$ \\
Sem evidência de leucemia & $415(91 \%)$ & $61(34 \%)$ & $16(7 \%$ \\
Retorno à fase crônica & $22(12 \%)$ & $7(3 \%)$ & \\
Resposta Citogenética Maior & $42(23 \%)$ & $43(19 \%)$ & \\
Completa & $248(55 \%)$ & $43(24 \%)$ & $36(16 \%)$ \\
Parcial & $164(36 \%)$ & $30(17 \%)$ & $15(7 \%)$ \\
\hline
\end{tabular}

Fonte: Sawyers CL; Capdeville R. Clinical Development on STI-571 in chronic myelo-genous leukemia. American Society of Hematology Educational Book 2001, p. 87-91 de pacientes que atingem respostas moleculares maiores e completas. A boa tolerabilidade desta medicação também é evidente no estudo citado. Os efeitos adversos mais freqüentes (náusea, edema, câimbras) são leves e, na maior parte das vezes, autolimitados. $^{7}$

Um subestudo do IRIS avaliou a resposta molecular destes pacientes através da quantificação de transcritos do gene híbrido BCR-ABL pelo método de reação de cadeia de polimerase em tempo real, sendo os resultados expressos em forma de redução logaritmica.

em fase crônica resistentes ou intolerantes ao alfa-interferon (STI-571 110), pacientes em fase acelerada (STI-571 109) e pacientes em crise blástica (STI-571 102). Os resultados destes estudos estão expostos de forma resumida na Tabela 1 .

\section{Tratamento inicial da LMC com mesilato de Imatinibe}

O estudo IRIS (International Randomized Study of Interferon and STI-571) é um estudo de fase III que compara interferon associado a citarabina em baixa dose, ao mesilato de imatinibe, em pacientes com leucemia mielóide crônica recém-diagnosticados. Um total de 1.106 pacientes foi randomizado, sendo 533 para receber imatinibe e 553 para receber interferon + citarabina.

Após um seguimento mediano de 19 meses, a taxa estimada de resposta citogenética maior aos 18 meses era de $87,1 \%$ para o grupo do imatinibe e de $34,7 \%$ no grupo que recebeu interferon e citarabina ( $\mathrm{p}<0,001)$. As taxas estimadas de resposta citogenética completa foram de 76,2\% para o imatinibe e $14,5 \%$ para o interferon e citarabina $(p<0,001)$. Aos 18 meses, a sobrevida livre de progressão para fase acelerada ou crise blástica foi de $96,7 \%$ e $91,5 \%$ respectivamente $(\mathrm{p}<0,001)$.

Um total de 79 pacientes (14,3\%) no grupo do imatinibe e 493 pacientes $(89,2 \%)$ no grupo do interferon e citarabina descontinuou ou mudou o tratamento inicial, devido a maior freqüência de eventos adversos grau três e quatro. Assim, o imatinibe foi superior à combinação de interferon-alfa e citarabina quanto à resposta hematológica, citogenética, tolerabilidade e probabilidade de progressão para fase acelerada e crise blástica em pacientes com leucemia mielóide crônica recém-diagnosticada. ${ }^{6}$

Na atualização de cinco anos deste estudo, o imatinibe foi capaz de induzir respostas citogenéticas completas em 87\% dos pacientes e uma sobrevida livre de progressão de 93\%. Além disso, há um crescente aumento no percentual
Após 12 meses, em pacientes com resposta citogenética completa houve redução de pelo menos 3 logs no nível de transcritos em 57\% dos pacientes com imatinibe e $29 \%$ dos pacientes com interferon $(\mathrm{P}=0.003)$. Para pacientes com remissão citogenética completa e uma redução de transcritos de pelo menos 3 logs em 12 meses, a probabilidade de permanecer livre de progressão foi de $100 \%$ aos 24 meses, comparada com 95\% para pacientes em remissão citogenética com redução menor de 3 logs e 85\% para pacientes que não alcançaram resposta citogenética completa aos 12 meses $(\mathrm{P}<0.001)$. Foi então definido o termo resposta molecular maior como a redução de 3 logs no nível de transcritos do gene BCR-ABL, e, como exposto, este nível de redução foi associado a uma sobrevida livre de progressão significativamente melhor. ${ }^{8}$

Quando avaliados quanto à qualidade de vida, os pacientes incluídos no estudo IRIS que foram randomizados para imatinibe tiveram mantidos o índice de função física e bem-estar, enquanto este foi substancialmente reduzido pelo tratamento com interferon e ara-C. Os escores médios de bem-estar familiar, social e emocional, assim como o escore de utilidade, também foram significativamente melhores nos pacientes que receberam imatinibe. Pacientes que cruzaram para o braço do imatinibe após intolerância ao IFN + Ara-C tiveram uma importante melhora nos índices citados. ${ }^{9}$

Apesar dos melhores resultados com imatinibe, no estudo IRIS não pode ser claramente demonstrada uma vantagem de sobrevida para estes pacientes quando comparados aos que receberam interferon + ara-C. No entanto, este fato se explica porque mais de $90 \%$ dos pacientes em uso de interferon foram posteriormente trocados para o braço do imatinibe. Alguns estudos compararam, no entanto, o uso de imatinibe com séries históricas de pacientes que usaram interferon por um período de tempo semelhante e, considerando-se as limitações deste tipo de comparação, o benefício de sobrevida em favor do imatinibe pode ser demonstrado. ${ }^{10,11}$ 
Ainda há questionamentos sobre a melhor dose de imatinibe a ser utilizada. Investigadores do MDACC reportaram resultados de um estudo comparativo entre pacientes com LMC recém-diagnosticada, tratados com 400 e $800 \mathrm{mg}$ diários de imatinibe. Em seis meses de seguimento, a taxa de resposta Tabela 2. Estudos com imatinibe como tratamento inicial

\begin{tabular}{lccc}
\hline Resposta & $\begin{array}{c}\text { IRIS } \\
(400 \mathrm{mg}) \mathrm{n} 1106\end{array}$ & $\begin{array}{c}\text { MD. ANDERSON } \\
(800 \mathrm{mg}) \mathrm{n}=114\end{array}$ & $\begin{array}{c}\text { TIDEL } \\
(600 \mathrm{mg})\end{array}=103$ \\
\hline Resposta Citogenética Maior & $92 \%$ & $95 \%$ & $94,2 \%$ \\
Completa & $87 \%$ & $90,3 \%$ & $88,5 \%$ \\
Resposta molecular maior & $80 \%^{\star}$ & $63 \%$ & $47,4 \%$ \\
Seguimento mediano & 60 meses & 15 meses & 12 meses \\
\hline
\end{tabular}

* Aos 12 meses 53\% citogenética completa foi de $52 \%$ versus $65 \%$ respectivamente. Um maior número de respostas moleculares foi ainda encontrado no grupo que fez uso de $800 \mathrm{mg}$ por dia desta medicação. ${ }^{9}$

Num outro estudo, investigadores da mesma instituição reportaram seus resultados com o tratamento de 114 pacientes com LMC recém-diagnosticada com mesilato de imatinibe na dose de $800 \mathrm{mg}$ ao dia. Cento e nove pacientes (95\%) tiveram resposta citogenética maior, sendo completa em 103 pacientes. Após um seguimento médio de 15 meses, nenhum paciente progrediu para fase acelerada ou crise blástica. A sobrevida estimada em dois anos foi de $94 \%$. Além disso, 71 (63\%) de 112 pacientes demonstraram redução da razão BCR-ABL/ABL para menos de 0,05\%, e em 31 pacientes (28\%) não foram detectados transcritos. Comparada à dose habitual de imatinibe, a dose de $800 \mathrm{mg}$ foi associada a uma melhor taxa de resposta citogenética completa $(\mathrm{P}=.0005)$, resposta molecular maior (QPRC $<0.05 \%$; $\mathrm{P}=.00001$ ), e resposta molecular completa (BCR-ABL indetectável; $\mathrm{P}=.001)$. A dose alta de imatinibe resultou em uma maior freqüência de mielossupressão, entretanto $82 \%$ dos pacientes continuaram a receber uma dose diária de 600 mg ou mais. ${ }^{10}$

O grupo australiano reportou resultados preliminares de um estudo de fase II (TIDEL) usando uma dose inicial de 600 mg de imatinibe, aumentada para 800 mg se critérios específicos de resposta não fossem alcançados: resposta hematológica completa aos três meses, resposta citogenética maior aos seis meses, resposta citogenética completa aos nove meses e redução de BCR-ABL/BCR (\%) maior que 4 logs aos 12 meses.

Dos 103 pacientes incluídos, 80 puderam ser avaliados aos 12 meses. A taxa de resposta citogenética maior foi de $94,2 \%$, com $88,5 \%$ de resposta citogenética completa e um índice de resposta molecular maior de $47,4 \%$. As taxas de RCM e RCC foram significativamente maiores quando comparadas aos resultados dos pacientes incluídos no estudo IRIS. Os pacientes que receberam dose diária menor que 600 mg nos primeiros dois meses tiveram uma taxa de RCC de $78 \%$, significativamente menor que a taxa de $93 \%$ alcançada em pacientes que receberam $600 \mathrm{mg}$ ao dia. A probabilidade de alcançar uma resposta molecular maior em seis meses foi de $58 \%$ para os pacientes que receberam uma dose média diária (DMM) de 600 mg e 33\% em pacientes com DMM de $500-599 \mathrm{mg}$ e $32 \%$ para aqueles com DMM $<500 \mathrm{mg}$. Os

grupos não eram significativamente diferentes quanto ao escore de Sokal. ${ }^{11}$

Apesar das melhores e mais precoces taxas de resposta citogenética e molecular (Tabela 2), um benefício claro de sobrevida ainda não pode ser demonstrado. Há estudos prospectivos em andamento para comparação entre o uso inicial de $400 \mathrm{mg}$ ou $800 \mathrm{mg}$ de imatinibe e o uso de inibidores como nilotinibe e dasatinibe. Estes inibidores tiveram ótimos resultados e boa tolerabilidade em fases mais tardias da doença, fato que motivou estes estudos. ${ }^{12,13}$ Até o momento, a dose inicial de $400 \mathrm{mg}$ de imatinibe é a dose recomendada para pacientes com LMC em fase crônica recém-diagnosticada. ${ }^{14}$

A despeito destes excelentes resultados, cerca de $28 \%$ dos pacientes do estudo IRIS descontinuaram o tratamento com imatinibe, uma parcela destes por intolerância ou falta de eficácia. Algumas características clínicas ou laboratoriais têm sido utilizadas para predizer a resposta individual de pacientes com o imatinibe. Pacientes com alto índice de Sokal têm menor probabilidade de resposta ao imatinibe. Alguns padrões de expressão genética também foram associados a diferentes respostas clínicas. Mais recentemente, a expressão do gene OCT- 1 foi identificada como um fator importante para a absorção do imatinibe e técnicas como a medida do IC50 também se correlacionaram com respostas clínicas. ${ }^{6,16,17}$

\section{Discussão}

A introdução do mesilato de imatinibe mudou o panorama do tratamento da Leucemia Mielóide Crônica em todo o mundo. O mesilato de imatinibe é considerado o tratamento padrão de primeira linha em pacientes com leucemia mielóide crônica recém-diagnosticados, proposição baseada nos excelentes resultados do estudo IRIS, cuja atualização de cinco anos demonstra uma sobrevida livre de progressão de $98 \%$ em pacientes que atingiram resposta citogenética completa e de $100 \%$ em pacientes com resposta molecular maior. Estes números são melhores que quaisquer resultados relatados com transplante de células-tronco hematopoiéticas (TCTH) até o momento, ${ }^{18,19,20} \mathrm{e}$ a toxicidade do medicamento é baixa, permitindo uma boa qualidade de vida aos pacientes, além do controle em longo prazo de sua enfermidade.

No entanto, alguns questionamentos permanecem em relação a este tratamento. Os efeitos desta droga em longo 
prazo ainda estão por ser definidos. O desenvolvimento de resistência devido a mutações, dentre estas a mutação T315, resistente a todos os inibidores disponíveis atualmente, considerações sobre a monitoração dos pacientes e o que deve ser feito em caso de resposta subótima, que dose inicial do medicamento deve ser utilizada (400 ou $800 \mathrm{mg}$ ), considerações sobre a aderência ao tratamento, cada vez mais importante no contexto de uma droga que tem que ser utilizada cronicamente, e por fim a preocupação acerca do custo da utilização vitalícia deste medicamento por uma população crescente de pacientes, todos estes são questionamentos ainda pertinentes. ${ }^{7}$

Apesar de todas estas considerações, os resultados do uso do mesilato de imatinibe em pacientes com LMC recém-diagnosticados e a excelente sobrevida livre de progressão alcançada, superior a qualquer outra intervenção terapêutica, estabeleceram esta droga como o tratamento de escolha para esta enfermidade em todo o mundo. Após cinco anos, o número de eventos relacionados à doença permanece em redução, consolidando a eficácia e segurança desta medicação. ${ }^{14}$

Infelizmente, apesar de todas estas evidências e contrariamente ao que ocorre na maioria dos países do mundo, o mesilato de imatinibe ainda não está disponível no Sistema Único de Saúde brasileiro para uso em primeira linha, fato que reduz a eficácia deste tratamento em nossos pacientes. O uso em fase mais tardias da doença aumenta a probabilidade de desenvolvimento de resistência e, conseqüentemente, há maior risco de progressão para fase acelerada e crise blástica, de evolução fatal. Há, portanto, urgência na modificação desta regulamentação a fim de que a nossa população possa receber este medicamento na sua melhor indicação.

\begin{abstract}
Imatinib mesylate is currently the gold-standard therapy for patients with newly diagnosed Chronic Myelogenous Leukemia. From the clinical trials in 1998 to the IRIS study, which compared first line imatinib treatment with interferon and low dose ara-C, this drug has been consolidated in regards to its safety and efficacy. There are still some questions to answer. Which would be the best initial dose? Are there any patients who benefit more than others? What is the best approach to suboptimal response and resistance? The most important published clinical studies are reviewed in the current article and discussed from a Brazilian perspective. Rev. bras. hematol. hemoter. 2008; 30(Supl.1):27-31.
\end{abstract}

Key words: Chronic Myeloid Leukemia; imatinib mesylate.

\section{Referências Bibliográficas}

1. Mauro MJ, O'Dwyer M, Heinrich MC, Druker BJ. STI-571: A paradigm of new agents for cancer therapeutics. J Clin Oncol. 2002;20(1):325-34.

2. Druker BJ, Talpaz M, Resta DJ, Peng B, Buchdunger E, Ford JM et al. Efficacy and safety of a specific inhibitor of the BCR-ABL tyrosine kinase in chronic myeloid leukemia. N Engl J Med. 2001; 5;344(14):1031-7.

3. Kantarjian HM, Sawyers C, Hochhaus A, Guillot F, Schiffer C, Gambacorti-Passerini C et al. Hematologic and cytogenetic responses to imatinib mesylate in chronic myelogenous leukemia. N Engl J Med 2002;346(9):645-52.

4. Talpaz M, Silver RT, Druker BJ, Goldman JM, Gambacorti-Passerini $\mathrm{C}$, Guillot $\mathrm{F}$ et al. Imatinib induces durable hematologic and cytogenetic responses in patients with accelerated phase chronic myeloid leukemia: results of a phase 2 study. Blood. 2002; 99(6): 1928-37.

5. Sawyers CL, Hochhaus A, Feldman E, Goldman JM, Miller CB, Ottmann OG et al. Imatinib induces hematologic and cytogenetic responses in patients with chronic myelogenous leukemia in myeloid blast crisis: results of a phase II study. Blood. 2002;99(10): 3530-9.

6. O'Brien SG, Guillot F, Larson RA, Gathmann I, Baccarani M, Cervantes $\mathrm{F}$ et al. Imatinib compared with interferon and lowdose cytarabine for newly diagnosed chronic-phase chronic myeloid leukemia. N Engl J Med. 2003;348(11):994-1004.

7. Druker BJ, Guillot F, O'Brien SG, Gathmann I, Kantarjian H, Gattermann $\mathrm{N}$ et al. Five-year follow-up of patients receiving imatinib for chronic myeloid leukemia. N Engl J Med. 2006;7; 355(23):2408-17.

8. Hughes TP, Kaeda J, Branford S, Rudzki Z, Hochhaus A, Hensley ML et al. Frequency of major molecular responses to imatinib or interferon alfa plus cytarabine in newly diagnosed chronic myeloid leukemia. N Engl J Med. 2003;349(15):1423-32.

9. Hahn EA, Glendenning GA, Sorensen MV, Druker BJ, Guillot F, Larson RA et al. Quality of life in patients with newly diagnosed chronic phase chronic myeloid leukemia on imatinib versus interferon alfa plus low-dose cytarabine: results from the IRIS Study. J Clin Oncol. 2003;21(11):2138-46.

10. Kantarjian HM, Talpaz M, O'Brien S, Jones D, Giles F, GarciaManero $G$ et al. Survival benefit with imatinib mesylate versus interferon-alpha-based regimens in newly diagnosed chronicphase chronic myelogenous leukemia. Blood. 2006; 108(6): 1835-40.

11. Hughes T, Branford S, Reynolds J, Seymour J, Taylor K, GuzzoPernell $\mathrm{N}$ et al. Higher-dose imatinib (600 mg/day) with selective intensification in newly diagnosed CML patients in chronic phase; cytogenetic response rates at 12 months are superior to IRIS. Blood (ASH Annual Meeting Abstracts) 2004; 104 (11):1001.

12. Kantarjian HM, Giles F, Gattermann, Bhalla K, Alimena G, Palandri F et al. Nilotinib (formerly AMN107), a highly selective BCRABL tyrosine kinase inhibitor, is effective in patients with Philadelphia chromosome-positive chronic myelogenous leukemia in chronic phase following imatinib resistance and intolerance. Blood. 2007;110(10):3540-46.

13. Kantarjian H, Pasquini R, Hamerschlak N, Rousselot P, Holowiecki $\mathrm{J}$, Jootar $\mathrm{S}$ et al. Dasatinib or high-dose imatinib for chronic-phase chronic myeloid leukemia after failure of first-line imatinib: a randomized phase 2 trial. Blood. 2007;109(12):5143-50.

14. Goldman JM. How I treat chronic myeloid leukemia in the imatinib era. Blood. 2007;110(8):2828-37.

15. Yong AS, Szydlo RM, Goldman JM, Apperley JF, Melo JV. Molecular profiling of CD34+ cells identifies low expression of 
CD7, along with high expression of proteinase 3 or elastase, as predictors of longer survival in patients with CML. Blood. 2006; 107(1):205-12.

16. White DL, Saunders VA, Dang P, Engler J, Zannettino AC, Cambareri AC et al.OCT-1-mediated influx is a key determinant of the intracellular uptake of imatinib but not nilotinib (AMN107): reduced OCT-1 activity is the cause of low in vitro sensitivity to imatinib. Blood. 2006;108(2):697-704.

17. White D, Saunders V, Lyons AB, Branford S, Grigg A, To LB et al. In vitro sensitivity to imatinib-induced inhibition of ABL kinase activity is predictive of molecular response in patients with de novo CML. Blood. 2005;106(7):2520-26.

18. Moreira VA, Zanis Neto J, Medeiros CR, Pasquini R, Bitencourt $\mathrm{M}$, Sakae $\mathrm{V}$ et al. Risk factor analysis of survival and graft versus host disease in patients with chronic myelogenous leukemia after allogeneic bone marrow transplantation. Blood (ASH Annual Meeting Abstracts)1999;94(1):10.

19. Horowitz MM, Rowlings PA, Passweg JR. Allogeneic bone marrow transplantation for CML: a report from the International Bone Marrow Transplant Registry. Bone Marrow Transplantation 1996; 17 suppl 3: 5-6.

20. Gratwohl A., Hermans, J. Allogeneic bone marrow transplantation for chronic myeloid leukemia. Bone Marrow Transplantation 1996;17 suppl 3: 7-9.

O tema apresentado e o convite ao autor constam da pauta elaborada pelos co-editores, Professor Ricardo Pasquini e

Professor Cármino Antonio de Souza.

Avaliação: Co-editores e um revisor externo.

Publicado após revisão e concordância do editor.

Conflito de interesse: não declarado.

Recebido: 21/2/2008

Aceito: 28/03/2008 